\title{
CARACTERIZACIÓN DEL RENDIMIENTO DE FORRAJE DE UNA PRADERA DE ALFALFA-OVILLO AL VARIAR LA FRECUENCIA E INTENSIDAD DEL PASTOREO
}

\author{
CHARACTERIZATION OF FORAGE YIELD OF AN ALFALFA-ORCHARDGRASS \\ PASTURE AT DIFFERENT GRAZING FREQUENCIES AND INTENSITIES
}

\author{
Alfonso Hernández Garay ${ }^{1}$, Pedro A. Martínez Hernández ${ }^{2 *}$, Job Zaragoza Esparza ${ }^{3}$, Humberto Vaquera \\ Huerta $^{4}$, Fernando Osnaya Gallardo ${ }^{3}$, Bertín M. Joaquín Torres ${ }^{1}$ y Ma. Eugenia Velazco Zebadúa ${ }^{5}$
}

\begin{abstract}
${ }^{1}$ Postgrado en Recursos Genéticos y Productividad-Ganadería y ${ }^{4}$ Postgrado en Estadística, Colegio de Postgraduados-Campus Montecillo. Km. 36.5 Carretera Federal México-Texcoco. 56230, Montecillo, Texcoco, Edo. de México. ${ }^{2}$ Programa de Posgrado en Producción Animal, Depto. de Zootecnia, Universidad Autónoma Chapingo. Km. 38.5 Carretera Federal México-Texcoco. 56230, Texcoco. ${ }^{3}$ Facultad de Estudios Superiores Cuautitlán, Universidad Nacional Autónoma de México. Carr. Cuautitla-Teoloyucan Km 2.5. San Sebastián Xhala. 54750, Cuautitlan Izcalli, Estado de México. ${ }^{5}$ Facultad de Medicina Veterinaria, Universidad Autónoma de Chiapas. Boulevard Belisario Domínguez 1081, 29050, Tuxtla Gutiérrez, Chiapas, México.
\end{abstract}

${ }^{*}$ Autor para correspondencia (pedroarturo@correo.chapingo.mx)

\section{RESUMEN}

Con la finalidad de determinar durante un año el comportamiento del rendimiento de forraje en una pradera de alfalfa-ovillo (Medicago sativa L.-Dactylis glomerata $\mathrm{L}$.) al variar la frecuencia e intensidad de pastoreo, se evaluaron seis tratamientos resultantes de la combinación factorial de dos frecuencias (alta y baja) y tres intensidades de pastoreo (ligera, media y severa, con alturas de forraje residual de 3 a 6, 7 a 10 y 11 a $14 \mathrm{~cm}$, respectivamente), en un diseño de bloques al azar con cuatro repeticiones. La unidad experimental fue una parcela de 100 $\mathrm{m}^{2}$. Para el pastoreo se emplearon borregos 'Columbia', sin ser sujetos de medición alguna. La interacción de los factores no mostró efecto en variable alguna. El rendimiento anual de forraje fue mayor $(4 \%)$ con alta frecuencia de pastoreo y el pastoreo severo $(3 \mathrm{a} 6 \mathrm{~cm})$ generó $19 \%$ más rendimiento anual de forraje. Los pastoreos frecuentes y severos favorecieron un mayor rendimiento de forraje de la pradera alfalfaovillo, pero también permitieron la invasión por malezas lo que puede reducir la persistencia de la pradera. El patrón de respuesta de la alfalfa en producción de forraje a los cambios en la intensidad de pastoreo fue diferente al del ovillo.

Palabras clave: Dactylis glomerata, Medicago sativa, composición botánica, pradera asociada, rendimiento de forraje, tasa de acumulación de forraje.

\section{SUMMARY}

In order to determine year-long forage yield performance of an alfalfa-orchardgrass (Medicago sativa L.-Dactylis glomerata L.) pasture subjected to different grazing frequencies and intensities, a $2 \times 3$ factorial arrangement of treatments (two frequencies, high and low) and three grazing intensities (severe, medium and light, with residual stubble height of 3 to 6,7 to $10 \mathrm{y} 11$ to $14 \mathrm{~cm}$, respectively), was evaluated under a block design with four replications in $100 \mathrm{~m}^{2}$ plots as experimental units. 'Columbia' sheep grazed freely and no data was collected from them. The interaction among factors was not significant in any of the measured variables. Annual forage yield was higher (4\%) with the high grazing frequency, and severe grazing $(3$ to $6 \mathrm{~cm})$ yielded more annual forage (19\%). Under frequent and severe grazing, the associated prairie can produce a higher forage yield along the year but with higher risk of weed infestation and decreased persistence. Alfalfa and orchardgrass species showed a different forage yield pattern in response to grazing intensity.

Index words: Dactylis glomerata, Medicago sativa, botanical composition, mixed pasture, forage yield, forage accumulation rate.

\section{INTRODUCCIÓN}

La frecuencia e intensidad de pastoreo pueden definir el nivel de producción animal de una pradera asociada porque las especies que la componen determinan la producción de forraje y su calidad, tanto en el aporte de forraje de cada especie como de cada componente morfológico (tallo, hoja y material muerto) presentes en la pradera (White y Hodgson, 1999). Además, el rendimiento de forraje de la pradera puede ser distinto si la cosecha se hace por pastoreo o en forma mecánica (Counce et al., 1984). La pradera asociada de alfalfa (Medicago sativa L.) y ovillo (Dactylis glomerata L.) se ha señalado como una de las mejores opciones para la producción bovina en pastoreo en algunas zonas templadas de México (Améndola et al., 1997).

El aporte que genera cada especie en una asociación vegetal a la biomasa total depende de la respuesta de cada una a los factores ambientales (Wakimoto et al., 1985). En una pradera asociada de alfalfa y raigrás perenne (Lolium perenne L.), la alfalfa presentó mayor velocidad de crecimiento y mayor aporte $(82 \%)$ al rendimiento debido al sombreado que ejerióe ejerció sobre el raigrás perenne; sin embargo, cuando se redujo la frecuencia de cosecha en la pradera se incrementó el rendimiento de forraje (Jung et al., 1996). Similarmente, la reducción de la intensidad de pastoreo, que involucra la reducción de la fotosíntesis residual y de los puntos de crecimiento, puede favorecer el rendimiento de la gramínea con relación a la alfalfa (Carlassare y Karsten, 2002). 
Debido a lo anterior y a la escasa la información sobre el comportamiento del rendimiento anual de forraje al aplicar diferentes frecuencias e intensidades de pastoreo, el objetivo de este estudio fue determinar, durante todo un año, el comportamiento del rendimiento de forraje de una pradera de alfalfa-ovillo al variar la frecuencia e intensidad de pastoreo.

\section{MATERIALES Y MÉTODOS}

La fase de campo se llevó a cabo en Cuautitlán, Estado de México, en un sitio ubicado a 19 43’ LN; 99 14’ LO y 2450 msnm, cuyo clima es templado, con 15.7 oC de temperatura media anual. La época fría del año se presenta en otoño e invierno (octubre a marzo), en la cual pueden presentarse temperaturas ambientales de 0 oC o menores; la época cálida del año se presenta en primavera y verano (abril a septiembre). La precipitación anual es de $664 \mathrm{~mm}$ y $80 \%$ de ella ocurre de junio a septiembre (García, 1988).

Se evaluaron seis tratamientos resultantes de la combinación factorial de dos frecuencias y tres intensidades de pastoreo (Cuadro 1).

Durante la época fría el intervalo entre pastoreos fue mayor porque la alfalfa y el ovillo redujeron sus tasas de crecimiento (Hernández et al., 1992). El diseño experimental fue bloques al azar con cuatro repeticiones y la unidad experimental fue una parcela de $100 \mathrm{~m}^{2}$. En febrero de $2002 \mathrm{se}$ sembraron las variedades comerciales de alfalfa ('Cuf-101') y de ovillo ('Potomac'). Con excepción de la época de mayor precipitación (junio a septiembre), cada $15 \mathrm{~d}$ se regó por aspersión con una lámina de $5 \mathrm{~cm}$. Se aplicaron tres cortes de formación para controlar malezas, el último de los cuales fue el 26 de junio, y 28 ó 35 d después, según la frecuencia de pastoreo, se aplicó el primer pastoreo experimental. Los pastoreos se mantuvieron hasta el 3 de julio de 2003, para cubrir un año continuo de aprovechamiento de la pradera.
Para introducir el efecto del pastoreo se aplicó la técnica de "pastoreo en masa" descrita por 't Mannetje et al. (1976), con borregos de la raza 'Columbia' de aproximadamente 60 $\mathrm{kg}$ de peso, y no se midió variable alguna en los borregos. Los pastoreos fueron por bloque; es decir, todas las parcelas dentro de un mismo bloque se pastorearon en un mismo día.

El rendimiento de forraje se expresó por año y por cada estación (verano, otoño, invierno y primavera). En verano y primavera se aplicó el intervalo entre pastoreos de la época cálida, mientras que en otoño e invierno se aplicó el correspondiente a la época fría. En cada pastoreo el rendimiento de forraje fue la diferencia de la masa de forraje al inicio del pastoreo menos la masa de forraje residual del pastoreo inmediato anterior. Ambas masas de forraje se estimaron mediante la cosecha a ras de suelo de todo el forraje presente en dos cuadrados de $0.25 \mathrm{~m}^{2}$ cada uno. En cada parcela estos cuadrados se colocaron en una trayectoria definida, para que durante el periodo experimental no se cosechara una misma área y que las áreas cosechadas para estimar las masas de forraje inicial y residual de un mismo periodo de pastoreo quedaran próximas entre sí.

El forraje cosechado de los cuadrados se lavó y secó en una estufa de aire forzado a $55^{\circ} \mathrm{C}$ hasta peso constante, para expresar el rendimiento con base en peso seco. El rendimiento anual de forraje fue la sumatoria de los rendimientos de forraje de todos los pastoreos realizados en cada tratamiento. El rendimiento de forraje por estación fue la sumatoria de los rendimientos de los pastoreos hechos en la estación respectiva; en esta sumatoria se incluyó el rendimiento de forraje del pastoreo de la estación siguiente si más de $50 \%$ del tiempo de descanso ocurrió en la estación considerada.

Con los datos del cociente entre el rendimiento de forraje y los días transcurridos desde el pastoreo próximo anterior,

Cuadro 1. Frecuencias e intensidades de pastoreo.

\begin{tabular}{llcc}
\hline \multirow{2}{*}{ Factor } & Nivel & \multicolumn{2}{c}{ Intervalos (días) entre pastoreos } \\
\cline { 3 - 4 } Frecuencia & Alta (AFP) & $\begin{array}{c}\text { Época cálida } \\
\text { (abril a septiembre) }\end{array}$ & $\begin{array}{c}\text { Época fría } \\
\text { (octubre a marzo) }\end{array}$ \\
& Baja (BFP) & 25 & 35 \\
& & 35 & 42 \\
\cline { 3 - 3 } Intensidad & Altura de forraje \\
& Ligera & residual (cm) & \\
& Media & 7 a 6 \\
& Severa & 11 a 14 \\
\hline
\end{tabular}


se calculó la tasa diaria de acumulación de forraje (TDAF). Además, del forraje cosechado para determinar masa de forraje al inicio del pastoreo efectuado hacia la mitad de cada estación, se tomó una muestra al azar de 250 a 300 g aproximadamente, que se separó en hoja y tallo de alfalfa u ovillo, material muerto de cualquiera de estas dos especies y otras especies (arvenses espontáneas, diferentes a ovillo o alfalfa), pues conforme se incrementa la invasión de especies no deseables es mayor el deterioro de la pradera. Cada componente se llevó a peso seco constante mediante la metodología antes descrita.

El análisis estadístico se basó en un análisis de varianza con una distribución de tratamientos en bloques al azar. Las variables analizadas fueron los rendimientos por año, por estación y dentro de cada estación por componente morfológico, material muerto y otras especies. La comparación de medias fue por medio de Tukey ( $\mathrm{P} \leq 0.05$; Steel y Torrie, 1988). Se utilizó el paquete PROC GLM de SAS (SAS Institute, 2001).

\section{RESULTADOS Y DISCUSIÓN}

\section{Rendimiento de forraje anual y por estación}

La frecuencia e intensidad de pastoreo tuvieron efecto en el rendimiento anual de forraje $(\mathrm{P}<0.05)$, pero no la interacción de ambos factores (Cuadro 2). El pastoreo menos frecuente y ligero registró menor rendimiento anual de forraje; i.e., la baja frecuencia de pastoreo (BFP) rindió $4 \%$ menos forraje que la alta (AFP), y el pastoreo ligero rindió $19 \%$ menos forraje que el severo. El incremento en la intensidad del pastoreo ocasionó una mayor diferencia en el rendimiento anual de forraje que el aumento en la frecuencia del pastoreo, lo que sugiere que dentro de los intervalos evaluados de frecuencia e intensidad de pastoreo, éste último factor fue el más importante durante el primer año de aprovechamiento de la pradera.

El menor rendimiento anual de forraje producido por la menor frecuencia de pastoreo implica que el mayor tiempo entre pastoreos consecutivos, no se reflejó en una acumulación de forraje que compensara el menor número de pastoreos. El mayor rendimiento anual de forraje de las praderas sometidas a pastoreo severo puede explicarse por el rebrote de la alfalfa, especie en la que el nuevo crecimiento proviene de las yemas coronarias y no de las axilares presentes en el forraje residual (Skinner y Moore, 2007). De hecho, el sombreado producido por el forraje residual puede interferir con la rápida activación de las yemas coronarias (Mitchell y Nelson, 2007) y con ello reducir la velocidad de rebrote.

La frecuencia e intensidad del pastoreo afectaron $(\mathrm{P}<$ 0.05 ) el rendimiento de forraje en primavera, verano e invierno, pero no la interacción entre ellos. En otoño solamente la frecuencia mostró efecto $(\mathrm{P}>0.05)$ sobre el rendimiento de forraje (Cuadro 3).

En verano e invierno, la alta frecuencia de pastoreo superó en $30 \%$ al rendimiento de la baja frecuencia; sin embargo, en primavera y otoño la baja frecuencia superó a la alta en 14 y $27 \%$, respectivamente. En ambientes donde la pradera de alfalfa-ovillo se mantiene durante todo el año, el impacto de las diferentes frecuencias de pastoreo debe evaluarse a lo largo de todo el año pues lo que sucede en una estación del año puede ser diferente en las otras estaciones. Gooding y Frame (1997) y Volenec y Nelson (2007) señalaron que en praderas pastoreadas, para lograr el máximo rendimiento de forraje y mínima invasión por otras especies, la frecuencia de pastoreo debe ser flexible, lo que permite que las plantas respondan a las variaciones de las condiciones ambientales durante el año o la estación de crecimiento.

En primavera, verano e invierno el rendimiento de forraje siempre fue menor con el pastoreo ligero que con el severo. La diferencia relativa entre estas dos intensidades fue máxima en el verano, donde el pastoreo severo rindió $34 \%$ más forraje que el pastoreo ligero. De esta manera se destaca la importancia de aplicar un pastoreo de alta frecuencia y severo en esta estación del año.

Cuadro 2. Rendimiento anual de forraje ( $\mathrm{kg} \mathrm{MS} \mathrm{ha}^{-1}$ ) en una pradera de alfalfa-ovillo.

\begin{tabular}{lccc}
\hline \multirow{2}{*}{ Intensidad de pastoreo } & \multicolumn{3}{c}{ Frecuencia de pastoreo } \\
\cline { 2 - 4 } & Alta & Baja & Promedio \\
\hline Severa & 25813 & 24930 & $25371 \mathrm{a}$ \\
Media & 24488 & 22943 & $23715 \mathrm{~b}$ \\
Ligera & 20829 & 20214 & $20521 \mathrm{c}$ \\
Promedio & $23710 \mathrm{a}$ & $22695 \mathrm{~b}$ & \\
\hline
\end{tabular}

Promedios con una literal en común en la misma columna o hilera, no son diferentes (Tukey; 0.05). Sin efecto de interacción $(\mathrm{P}>0.05)$. 
Cuadro 3. Rendimiento de forraje (kg MS ha-1) por estación en una pradera de alfalfa-ovillo.

\begin{tabular}{|c|c|c|c|c|c|c|}
\hline \multirow{3}{*}{ Intensidad de pastoreo } & \multicolumn{6}{|c|}{ Estación del año } \\
\hline & \multicolumn{3}{|c|}{ Verano } & \multicolumn{3}{|c|}{ Otoño } \\
\hline & AFP & $\mathrm{BFP}$ & Promedio & AFP & $\mathrm{BFP}$ & Promedio \\
\hline Severa & 7606 & 5978 & $6792 \mathrm{a}$ & 2992 & 4453 & 3723 \\
\hline Media & 6923 & 5275 & $6099 \mathrm{~b}$ & 3606 & 4152 & 3879 \\
\hline Ligera & 5667 & 4436 & $5052 c$ & 3044 & 3595 & 3319 \\
\hline Promedio & $6732 \mathrm{a}$ & $5230 \mathrm{~b}$ & & $3214 b$ & $4067 \mathrm{a}$ & \\
\hline
\end{tabular}

\begin{tabular}{|c|c|c|c|c|c|c|}
\hline & \multicolumn{3}{|c|}{ Invierno } & \multicolumn{3}{|c|}{ Primavera } \\
\hline & AFP & BFP & Promedio & AFP & BFP & Promedio \\
\hline Severa & 6516 & 5422 & 5969 a & 8699 & 9077 & $8888 \mathrm{a}$ \\
\hline Media & 6700 & 4931 & $5816 a$ & 7260 & 8585 & 7922 b \\
\hline Ligera & 5616 & 4175 & $4896 \mathrm{~b}$ & 6502 & 8008 & $7255 \mathrm{c}$ \\
\hline Promedio & $6277 \mathrm{a}$ & $4843 \mathrm{~b}$ & & $7484 \mathrm{~b}$ & 8556 a & \\
\hline
\end{tabular}

\section{Tasa diaria de acumulación de forraje (TDAF)}

En ninguna estación del año la TDAF mostró efecto $(\mathrm{P}>$ $0.05)$ de la interacción frecuencia por intensidad de pastoreo; en primavera hubo efecto $(\mathrm{P}<0.05)$ de ambos factores; en invierno lo hubo de la frecuencia y de la intensidad en verano (Cuadro 4). La baja frecuencia de pastoreo mostró una TDAF 16 y $41 \%$ mayor que la alta frecuencia en primavera e invierno, respectivamente. La mayor TDAF de la baja frecuencia originó mayor rendimiento de forraje en primavera pero no en invierno (Cuadro 3), lo que indica que solamente cuando la TDAF era muy alta el rendimiento estacional de forraje dependía de este atributo; de lo contrario, el factor determinante era el número de pastoreos realizados.

El pastoreo severo registró una TDAF 32 y 73 \% superior a la del ligero en primavera y verano, respectivamente. Esto muestra que un componente de manejo para lograr máximos rendimientos de forraje en praderas de alfalfaovillo, es la aplicación de pastoreos severos porque favorece una mayor tasa diaria de acumulación de forraje debido al rebrote de la alfalfa proveniente de yemas coronarias, sin aporte al nuevo crecimiento de los tallos no cosechados (Mitchell y Nelson, 2007); en cambio, el rebrote en ovillo no necesariamente se promueve con el pastoreo severo, como mencionaron Carlassare y Karsten (2002).

\section{Rendimiento por componente morfológico}

En ninguna estación del año la interacción frecuencia por intensidad de pastoreo mostró efecto $(\mathrm{P}>0.05)$ en el rendimiento de cualquier órgano de la planta. En alfalfa, la frecuencia e intensidad de pastoreo tuvieron efecto sobre el rendimiento de hoja y tallo $(\mathrm{P}<0.05)$ en las cuatro estaciones. En ovillo, la frecuencia de pastoreo influyó $(\mathrm{P}<0.05)$ en los rendimientos de hoja y tallo en el verano, de hoja en primavera y del tallo en invierno. La intensidad de pastoreo no tuvo efecto $(\mathrm{P}>0.05)$ en el rendimiento de tallo durante la primavera (Cuadros 5 y 6 ).

Alfalfa y ovillo respondieron igual a la frecuencia de pastoreo al presentar mayores rendimientos de hoja y tallo en BFP que en AFP. Esta respuesta coincide con la observada en la TDAF donde la baja frecuencia también superó a la alta frecuencia de pastoreo (Cuadro 4). Por tanto, el mayor rendimiento anual de forraje encontrado con AFP (Cuadro 2) se atribuye al mayor número de pastoreos respecto a la BFP, ya que nunca superó a ésta en TDAF o en los rendimientos de sus componentes morfológicos.

El patrón de respuesta a la intensidad de pastoreo fue diferente en alfalfa y ovillo. En la primera, el pastoreo severo promovió mayor rendimiento que el pastoreo ligero, y en ovillo fue lo contario pues el pastoreo ligero superó al severo. La respuesta de alfalfa en rendimientos de hoja y tallo a la intensidad de pastoreo, fue igual a la del rendimiento de forraje y TDAF (Cuadros 2, 3 y 4), por lo que se considera que la alfalfa es la que determinó el patrón de respuesta a la severidad de pastoreo en la asociación alfalfa-ovillo. Jung et al. (1996) también indicaron que en praderas asociadas gramínea-leguminosa de zona templada, los componentes pueden mostrar respuestas contrastantes a las variaciones 
Cuadro 4. Tasa diaria de acumulación de forraje $\left(\mathrm{kg} \mathrm{MS} \mathrm{ha}^{-1}\right)$ en una pradera de alfalfa-ovillo.

\begin{tabular}{|c|c|c|c|c|c|c|}
\hline \multirow{3}{*}{ Intensidad de pastoreo } & \multicolumn{6}{|c|}{ Estación del año } \\
\hline & \multicolumn{3}{|c|}{ Verano } & \multicolumn{3}{|c|}{ Otoño } \\
\hline & AFP & $\mathrm{BFP}$ & Promedio & AFP & $\mathrm{BFP}$ & Promedio \\
\hline Severa & 94 & 96 & $95 \mathrm{a}$ & 76 & 74 & 75 \\
\hline Media & 77 & 80 & $78 \mathrm{~b}$ & 84 & 83 & 83 \\
\hline Ligera & 60 & 50 & $55 \mathrm{c}$ & 69 & 89 & 79 \\
\hline \multirow[t]{3}{*}{ Promedio } & 77 & 75 & & 77 & 82 & \\
\hline & \multicolumn{3}{|c|}{ Invierno } & \multicolumn{3}{|c|}{ Primavera } \\
\hline & AFP & $\mathrm{BFP}$ & Promedio & AFP & $\mathrm{BFP}$ & Promedio \\
\hline Severa & 41 & 48 & 44 & 109 & 105 & $107 \mathrm{a}$ \\
\hline Media & 42 & 63 & 52 & 77 & 99 & $88 \mathrm{~b}$ \\
\hline Ligera & 40 & 64 & 52 & 70 & 93 & $81 \mathrm{~b}$ \\
\hline Promedio & $41 \mathrm{~b}$ & $58 \mathrm{a}$ & & $85 \mathrm{~b}$ & $99 \mathrm{a}$ & \\
\hline
\end{tabular}

en la intensidad de cosecha, y que en asociaciones con alfalfa esta especie es la que determina el patrón de respuesta del rendimiento de forraje.

El mayor rendimiento que ovillo presentó con el pastoreo ligero, puede atribuirse a que en ese tipo de pastoreo las gramíneas adaptadas a climas templados quedan con hojas residuales capaces de fotosintetizar, lo que favorece un rebrote vigoroso (White y Hodgson, 1999). Al respecto, Carlassare y Karsten (2002) encontraron que el ovillo manejado con cosecha ligera mostró un rebrote más vigoroso y con mayor tasa diaria de acumulación, debido a la fotosíntesis de las hojas residuales.

Los mayores rendimientos de hoja y tallo con el pastoreo severo que la alfalfa presentó, pueden atribuirse a que los rebrotes de esta especie provienen de yemas coronarias y a que los tallos y hojas residuales no originan nuevo crecimiento ni hacen un aporte fotosintético importante al mismo (Moser y Jennings, 2007).

La frecuencia e intensidad de pastoreo en invierno, y la intensidad de pastoreo en primavera influyeron $(\mathrm{P}<0.05)$ en el rendimiento de otras especies (Cuadros 5 y 6). El aporte de otras especies fue 2.7 veces mayor en alta que en baja frecuencia de pastoreo, y en el pastoreo severo fue de 3 a 8 veces mayor que en el ligero. Esto significa que la persistencia de la pradera de alfalfa-ovillo con AFP y pastoreo severo podría estar en mayor riesgo, ya que según Smith et al. (2000) después de la muerte invernal es la invasión de especies espontáneas lo que reduce la permanencia de pra- deras de alfalfa, tanto en monocultivo como en asociación.

En otoño, el rendimiento de material muerto varió $(\mathrm{P}<$ $0.05)$ por la intensidad de pastoreo; en las otras estaciones influyeron la frecuencia e intensidad de pastoreo (Cuadros 5 y 6). En este caso la intensidad del pastoreo mostró un efecto más estable que la frecuencia; en todas las estaciones: el pastoreo ligero presentó entre 1.3 a 2.4 veces más material muerto que el pastoreo severo, posiblemente originado en su mayor parte del forraje residual de la alfalfa, ya que el rebrote de esta especie se deriva de las yemas coronarias mientras que el forraje residual entra en senescencia y muere (Moser y Jennings, 2007).

\section{CONCLUSIONES}

En una pradera de alfalfa-ovillo aprovechada por pastoreo, el rendimiento anual y por estación, así como la magnitud y duración de la tasa diaria de acumulación de forraje, dependen de la frecuencia e intensidad de pastoreo aplicados. El efecto de la frecuencia en el comportamiento de la pradera de alfalfa-ovillo es independiente del de la intensidad de pastoreo.

El pastoreo frecuente y severo de una pradera de alfalfaovillo favorece la invasión de la misma por especies ajenas a la asociación, lo que aumenta el riesgo de una menor persistencia de la pradera.

Alfalfa y ovillo mostraron un patrón contrastante de respuesta en el rendimiento de sus componentes morfológicos por efecto de la intensidad de pastoreo. 
Cuadro 5. Rendimiento (kg MS ha-1) por componente morfológico de cada especie en una pradera de alfalfa-ovillo en verano y otoño.

\begin{tabular}{|c|c|c|c|c|c|c|}
\hline \multirow{3}{*}{ Intensidad de pastoreo } & \multicolumn{6}{|c|}{ Estación del año } \\
\hline & \multicolumn{3}{|c|}{ Verano } & \multicolumn{3}{|c|}{ Otoño } \\
\hline & AFP & BFP & Promedio & AFP & BFP & Promedio \\
\hline & \multicolumn{6}{|c|}{ Hoja de alfalfa } \\
\hline Severa & 1001 & 1067 & $1034 \mathrm{a}$ & 863 & 926 & $894 \mathrm{a}$ \\
\hline Media & 706 & 859 & $782 \mathrm{~b}$ & 747 & 875 & $811 \mathrm{a}$ \\
\hline Ligera & 653 & 734 & $693 c$ & 444 & 617 & $531 \mathrm{~b}$ \\
\hline \multirow[t]{2}{*}{ Promedio } & $787 \mathrm{~b}$ & 887 a & & $684 \mathrm{~b}$ & $806 \mathrm{a}$ & \\
\hline & \multicolumn{6}{|c|}{ Tallo de alfalfa } \\
\hline Severa & 1066 & 1227 & $1146 \mathrm{a}$ & 1042 & 1157 & 1099 a \\
\hline Media & 821 & 1014 & $917 b$ & 996 & 1060 & $1028 \mathrm{a}$ \\
\hline Ligera & 715 & 858 & $787 c$ & 631 & 828 & $729 \mathrm{~b}$ \\
\hline \multirow[t]{2}{*}{ Promedio } & $867 \mathrm{~b}$ & $1033 \mathrm{a}$ & & $890 \mathrm{~b}$ & $1015 \mathrm{a}$ & \\
\hline & \multicolumn{6}{|c|}{ Hoja de ovillo } \\
\hline Severo & 455 & 966 & 711 & 115 & 203 & $159 \mathrm{c}$ \\
\hline Medio & 359 & 524 & 441 & 215 & 243 & $229 b$ \\
\hline Ligero & 419 & 617 & 518 & 281 & 270 & $276 \mathrm{a}$ \\
\hline \multirow[t]{2}{*}{ Promedio } & $411 \mathrm{~b}$ & $702 \mathrm{a}$ & & 204 & 239 & \\
\hline & \multicolumn{6}{|c|}{ Tallo de ovillo } \\
\hline Severo & 148 & 228 & 188 & 24 & 36 & $30 \mathrm{~b}$ \\
\hline Medio & 99 & 169 & 134 & 74 & 40 & $57 \mathrm{a}$ \\
\hline Ligero & 122 & 268 & 195 & 66 & 58 & $62 \mathrm{a}$ \\
\hline \multirow[t]{2}{*}{ Promedio } & $123 \mathrm{~b}$ & $222 \mathrm{a}$ & & 55 & 45 & \\
\hline & \multicolumn{6}{|c|}{ Otras especies } \\
\hline Severo & 99 & 123 & 111 & 0 & 0 & 0 \\
\hline Medio & 102 & 111 & 106 & 0 & 0 & 0 \\
\hline Ligero & 86 & 46 & 66 & 0 & 0 & 0 \\
\hline \multirow[t]{2}{*}{ Promedio } & 96 & 93 & & 0 & 0 & 0 \\
\hline & \multicolumn{6}{|c|}{ Material muerto } \\
\hline Severo & 81 & 60 & $71 \mathrm{~b}$ & 60 & 57 & $59 \mathrm{~b}$ \\
\hline Medio & 102 & 90 & $96 \mathrm{a}$ & 126 & 69 & $98 \mathrm{a}$ \\
\hline Ligero & 113 & 85 & 99 a & 98 & 118 & $108 \mathrm{a}$ \\
\hline Promedio & $99 \mathrm{a}$ & $78 \mathrm{~b}$ & & 95 & 81 & \\
\hline
\end{tabular}


Cuadro 6. Rendimiento $\left(\mathrm{kg} \mathrm{MS} \mathrm{ha}^{-1}\right)$ por componente morfológico de cada especie en una pradera de alfalfa-ovillo en invierno y primavera.

\begin{tabular}{|c|c|c|c|c|c|c|}
\hline \multirow{3}{*}{ Intensidad de pastoreo } & \multicolumn{6}{|c|}{ Estación del año } \\
\hline & \multicolumn{3}{|c|}{ Verano } & \multicolumn{3}{|c|}{ Otoño } \\
\hline & AFP & BFP & Promedio & AFP & BFP & Promedio \\
\hline & \multicolumn{6}{|c|}{ Hoja de alfalfa } \\
\hline Severo & 737 & 1099 & $918 \mathrm{a}$ & 666 & 919 & $792 \mathrm{a}$ \\
\hline Medio & 664 & 821 & $743 \mathrm{~b}$ & 506 & 772 & $639 \mathrm{~b}$ \\
\hline Ligero & 514 & 518 & $516 c$ & 455 & 694 & $574 \mathrm{~b}$ \\
\hline Promedio & $638 \mathrm{~b}$ & $813 a$ & & $542 \mathrm{~b}$ & $795 \mathrm{a}$ & \\
\hline
\end{tabular}

Tallo de alfalfa

$\begin{array}{lllllll}\text { Severo } & 682 & 1098 & 890 \mathrm{a} & 721 & 1017 & 869 \mathrm{a} \\ \text { Medio } & 594 & 949 & 771 \mathrm{~b} & 575 & 812 & 693 \mathrm{~b} \\ \text { Ligero } & 634 & 497 & 565 \mathrm{c} & 528 & 691 & 610 \mathrm{~b} \\ \text { Promedio } & 636 \mathrm{~b} & 848 \mathrm{a} & & 608 \mathrm{~b} & 840 \mathrm{a} & \end{array}$

Hoja de ovillo

$\begin{array}{lrrrlll}\text { Severo } & 83 & 90 & 87 \mathrm{~b} & 257 & 624 & 440 \mathrm{~b} \\ \text { Medio } & 94 & 122 & 108 \mathrm{~b} & 756 & 784 & 770 \mathrm{a} \\ \text { Ligero } & 161 & 162 & 162 \mathrm{a} & 692 & 807 & 750 \mathrm{a} \\ \text { Promedio } & 113 & 125 & & 569 \mathrm{~b} & 738 \mathrm{a} & \end{array}$

$\begin{array}{llllrrr}\text { Severo } & 9 & 14 & 12 \mathrm{~b} & 59 & 73 & 66 \\ \text { Medio } & 10 & 17 & 13 \mathrm{~b} & 125 & 97 & 111 \\ \text { Ligero } & 16 & 20 & 18 \mathrm{a} & 132 & 81 & 106 \\ \text { Promedio } & 12 \mathrm{~b} & 17 \mathrm{a} & & 105 & 84 & \end{array}$

\begin{tabular}{|c|c|c|c|c|c|c|}
\hline Severo & 48 & 18 & $33 \mathrm{a}$ & 125 & 149 & $137 \mathrm{a}$ \\
\hline Medio & 25 & 11 & $18 \mathrm{~b}$ & 87 & 124 & $105 \mathrm{a}$ \\
\hline Ligero & 8 & 1 & $4 \mathrm{c}$ & 61 & 39 & $50 \mathrm{~b}$ \\
\hline \multirow[t]{2}{*}{ Promedio } & $27 \mathrm{a}$ & $10 \mathrm{~b}$ & & 91 & 104 & \\
\hline & \multicolumn{6}{|c|}{ Material muerto } \\
\hline Severo & 104 & 191 & $147 \mathrm{c}$ & 93 & 264 & $178 \mathrm{c}$ \\
\hline Medio & 252 & 299 & $276 \mathrm{~b}$ & 178 & 435 & $307 \mathrm{~b}$ \\
\hline Ligero & 274 & 383 & $328 \mathrm{a}$ & 329 & 540 & $434 \mathrm{a}$ \\
\hline Promedio & $210 \mathrm{~b}$ & $291 \mathrm{a}$ & & $200 \mathrm{~b}$ & $413 \mathrm{a}$ & \\
\hline
\end{tabular}




\section{BIBLIOGRAFÍA}

Améndola M R, C A García, J R Juárez (1997) Grass-legume mixtures under grazing. In: Proc. XVIII International Grassland Congress. 11-16 july. Winnipeg and Saskatoon, Canada. pp:121122.

Carlassare M, H D Karsten (2002) Species population dynamics in a mixed pasture under two rotational sward grazing height regimes. Agron. J. 95:844 - 854.

Counce P A, J H Bouton, R H Brown (1984). Screening and characterizing alfalfa for persistence under mowing and continuous grazing. Crop Sci. 24:282 - 285.

García E (1988) Modificaciones al Sistema de Clasificación Climática de Köppen para Adaptarlo a la República Mexicana. Instituto de Geografía, Universidad Nacional Autónoma de México. México. 217 p.

Gooding R F, J Frame (1997) Effects of continuous sheep stocking and strategic rest periods on the sward of binary perennial grass/ white clover associations. Grass Forage Sci. 52:350 - 357.

Hernández G A, J Pérez, V A González (1992) Crecimiento y rendimiento de la alfalfa en respuesta a diferentes regímenes de cosecha. Agrociencia 2:131 - 144 .

Jung G A, J A Shaffer, J R Everhart (1996) Harvest frequency and cultivar influence on yield and protein of alfalfa-ryegrass mixtures. Agron. J. 88:817 - 822.

Mitchell R B, C J Nelson (2007) Structure and morphology of legumes and other forbs. In: Forages, an Introduction to Grassland Agriculture. Volume I. R F Barnes, C J Nelson, M Collins, K J Moore (eds). 6th ed. Blackwell Publishing, Ames, Iowa, USA. pp:51-72.
Moser L E, J A Jennings (2007) Grass and legume structure and morphology. In: Forages, the Science of Grassland Agriculture. Volume II. R F Barnes, C J Nelson, M Collins, K J Moore (eds). 6th ed. Blackwell Publishing. Ames, Iowa, USA. pp:15 - 36

SAS Institute (2001) SAS User's Guide. Release 8.1. 6th ed. SAS Institute, Inc. Cary, NC, USA. 956 p.

Skinner R H, K J Moore (2007) Growth and development of forage plants: In: Forages, the Science of Grassland Agriculture. Volume II. R F Barnes, C J Nelson, M Collins, K J Moore (eds). 6th ed. Blackwell Publishing. Ames, Iowa, USA. pp:53-66.

Smith S R Jr, J H Bouton, A Singh, W P McCaughey (2000) Development and evaluation of grazing-tolerant alfalfa cultivars: A review. Can. J. Plant Sci. 80:503-512.

Steel R G D, J H Torrie (1988) Bioestadística: Principios y Procedimientos. 2a ed. Mc Graw-Hill/Interamericana de México. México. $622 \mathrm{p}$.

't Mannetje L, R J Jones, T H Stobbs (1976) Pasture evaluation by grazing experiments. In: Tropical Pasture Research. Principles and Methods. N H Shaw, W W Bryan (eds). C. B. Pasture Field Crops. Bull. 51. Hurley, England. pp:194-234.

Volenec J J, C J Nelson (2007) Environmental aspects of forage management. In: Forages, an Introduction to Grassland Agriculture. Volume I. R F Barnes, C J Nelson, M Collins, K J Moore (eds). 6th ed. Blackwell Publishing. Ames, Iowa, USA. pp:99-124.

Wakimoto T, Y Stage, M Tagawa, T Kitamori. (1985) Interaction of component cultivars in grass-alfalfa mixtures. In: Proc. XV International Grassland Congress. 20-25 July. Kyoto, Japan. pp:682-684.

White J, J Hodgson (1999) New Zealand Pasture and Crop Science. Oxford University Press. Auckland, New Zealand. 323 p. 